\title{
DYNAMIC OBSTACLE AVOIDANCE AND TRAJECTORY PLANNING OF A FIVE AXIS REDUNDANT INDUSTRIAL MANIPULATOR
}

\author{
Yali Zhao ${ }^{*}$, Yamin Li ${ }^{1}$, Tiancong Chen ${ }^{1}$ \\ ${ }_{1}^{1}$ Mechanic and Electronic Engineering, Xinlian College of Henan Normal University, \\ Zhengzhou, 451400, Henan, China \\ *Corresponding author: Yali Zhao \\ Email: zhaoyalihnnu@yeah.net
}

\begin{abstract}
The dynamic obstacle avoidance of the five-axis redundant industrial manipulator is studied; its trajectory planning is established and simulation analysis is conducted. The five-axis redundant industrial manipulator is modelled first, and then its motion model is established by Denavit-Hartenberg. Based on the motion logic, the joint motion orders are analysed, and the motion model is made by algebraic method. Subsequently, the model is simplified and the obtained trajectory should conform to the requirements. Meantime, the arm plane and obstacle avoidance surface are introduced to parametrically express the zero-space motion of the redundant manipulator. In terms of the predetermined detection target, the artificial potential field method is used to detect the collision between the manipulator and the obstacle, and the motion process of the virtual repulsion force in space can be detected, improving the previous control method for inverse motion, which makes the dynamic performance of the manipulator have the similar physical properties of quasi mass-damping system in obstacle avoidance. This method can not only effectively control the end effector, but also realize the obstacle avoidance of the manipulator. To test feasibility of the proposed method, the experiment is conducted through the rail selfmaintenance. Through the simulation of dynamic obstacle avoidance and trajectory planning, it is found that the nearest distance between the manipulator and the obstacle is more than $40 \mathrm{~mm}$, the dynamic difference of the end effector is less than $9 \mathrm{~mm}$, and the static difference is less than $3 \mathrm{~mm}$. The self-motion of the manipulator is studied to address the problem of obstacle avoidance. This method has no effect on the end effector and provides a technical reference for the unmanned system driving.
\end{abstract}

Keywords: Manipulator; Artificial potential field method; Dynamic obstacle avoidance; Trajectory planning; Inverse dynamics.

\section{Introduction}

With the progress of science and technology, the development of robots, especially bionic robots, is particularly rapid. In some complex situations, when the bionic robot manipulator works, the safety problems caused by human-machine interaction attract the public. In the past, when the manipulator is about to collide with or has collided with personnel, the emergency stop will be used to avoid accidents. However, in the current complex interactive environment, this method may cause more harm to the manipulator and personnel [1,2]. Therefore, more advanced safety obstacle avoidance (also known as collision avoidance) methods need to be used to ensure the continuity of mechanical work.

Compared with other manipulators, redundant manipulators have more advantages because they can use redundant degrees of freedom to perform obstacle avoidance without affecting the end effector.
Global planning and local control are two kinds of methods used by redundant manipulators in obstacle avoidance. The first method is global planning, that is, the robot arm and obstacles are combined and projected into the $\mathrm{C}$ space (Configuration Space) to determine the unoccupied part of the space and find the collision-free route, which is similar to the upper path planning $[3,4]$. However, this method also has some defects. For example, the large amount of computation leads to more response time than the general manipulator, and the complexity shows that even if the algorithm can be optimized to reduce the amount of computation, it is difficult to apply to the actual obstacle avoidance. The second method is local control, which is different from global planning obstacle avoidance in that it is based on the control level and solves the obstacle avoidance problem through the bottom control, which involves a small amount of calculation and more flexible calculation 
[5]. The local control method is proposed for the redundant manipulator to perform obstacle avoidance, and the critical point in the obstacle avoidance is kept away from the obstacles. Some scholars use the method of the pseudo-inverse matrix to calculate the real-time distance between the arm and the obstacle. Based on the potential function, the change of the interaction force between the obstacle and the target position is calculated, and the objective function is upgraded and optimized to maximize the safe distance between the manipulator and the obstacle [6]. Nowadays, many control systems of manipulators still take obstacle avoidance as the main task in constructing a manipulator. And most methods are used to tackle the obstacle avoidance problem of manipulators following the kinematics. In fact, the problem can also be solved by the acceleration of the manipulator and joint torque, which can improve the performance of the manipulator.

Since the manipulator is the simulation of a human arm to realize movements, obstacle avoidance of the manipulator should be studied from the perspective of kinematics after the motion rule and gravity torque of the manipulator are calculated; and the problem of obstacle avoidance of the manipulator should be tackled following the dynamics, which can be combined with the control method [7].

The obstacle avoidance control method is a local control method combining dynamics and kinematics. The purpose is to solve the problem of obstacle avoidance and trajectory planning of redundant manipulators in dynamic control. To achieve obstacle avoidance, the manipulator can be regarded as a parameterized mass-damping system. When the external force is applied to the manipulator, it will move [8]. Hence, a kind of virtual force acting on the manipulator is needed to make the manipulator move automatically to achieve obstacle avoidance. In the process of completing the task, when the manipulator is close to the obstacle, the obstacle will send out a repulsive force, which makes the manipulator move automatically to avoid contact or collision with obstacles. However, the virtual repulsive force must be calculated accurately. Through the research on the model of the 7-DOF (Degree of Freedom) redundant manipulator, the second-order linear equation of the mass-damping system is established to improve and optimize the inverse kinematics control method. Thus, the manipulator realizes obstacle avoidance and trajectory planning in the process of executing the task, and the positioning and tracking of the end effector will not be affected [9-11].

\section{Manipulator Design and Experimental Platform Construction}

\subsection{Design of Redundant Industrial Manipulator}

In most cases, the non-redundant DOF Manipulator can meet the task requirements in the workspace, but it cannot avoid the singular positioning and obstacles in the task space. Because of the self-motion of the redundant manipulator, the singular configuration and obstacle avoidance in the workspace can be avoided. In addition, the motion flexibility of redundant manipulators can prevent movement overrun and improve dynamics and performance. Four standards for the structural design of redundant manipulator are summarized: (1) it can eliminate the singular position in the workspace, which is also a basis for structural design; (2) it optimizes the working space, that is, the increased degree of freedom can solve the obstacle avoidance problem as much as possible; (3) it can make kinematics calculation easy; (4) it can simplify the structure, that is, the increased joints must be reduced to the minimum compared with the original mechanism design [12].

The 7-DOF manipulator can meet the above four design standards before the redundant degree is selected. The super redundant manipulator with more degrees of freedom not only makes the kinematic calculation more complex, but also cannot completely solve the problem of internal singular configuration.

The design of the 7-DOF manipulator can be considered as adding one joint on the basis of the 6DOF manipulator. And the most important geometric configuration of the 6-DOF manipulator is the 7-axis manipulator, as shown in Figure 1. Compared with other 6-DOF manipulators, this kind of manipulator can adapt to the workspace quickly.

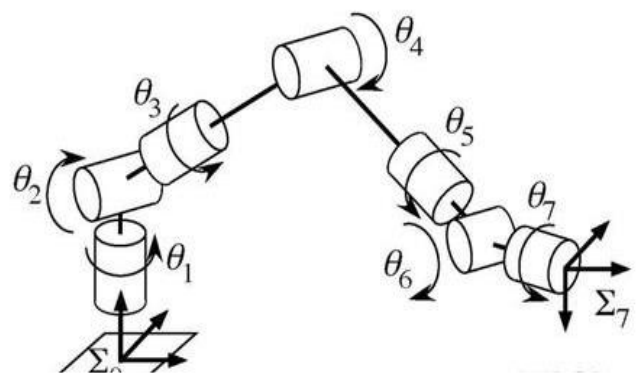

Figure. 1 Structure of 7-axis manipulator

Based on the structure of the 7-axis manipulator, the additional joint is finally cross-installed to the seventh joint at the shoulder joint of the manipulator, as shown in Figure 2.

This arrangement makes the shoulder joint have 7 DOF, and the joint arrangement of the whole manipulator is similar to that of the human arm [13]. 
This kind of degree of freedom can greatly simplify the kinematic calculation, and the mechanical design is relatively easy and popular.

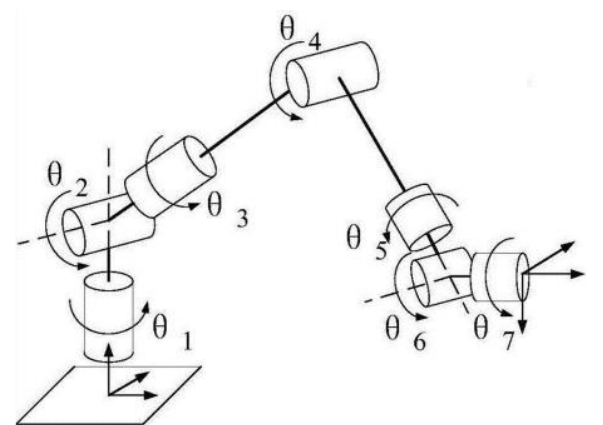

Figure 2 Structure configuration of the 7-DOF manipulator

\subsection{Manipulator Control in position inner loop}

For a manipulator with $\mathrm{n}$ degrees of freedom, the following dynamic equation is established for the contact force between the manipulator and the surroundings:

$$
M(\mathrm{q}) \ddot{q}+C(\mathrm{q}, \dot{q})+\mathrm{d}(\mathrm{q}, \dot{q})+\mathrm{G}(\mathrm{q})=\tau_{a}+\tau_{e x t}
$$

In the equation, $q \in \boldsymbol{R}^{\mathrm{n}}$ is the joint angle vector, $q \in R^{n}$ is the joint angular velocity vector, $\ddot{q} \in R^{n}$ is the acceleration vector in the process of joint angular motion, $\boldsymbol{M} \in \boldsymbol{R}^{\mathrm{n}^{* \mathrm{n}}}$ is the positive inertia matrix, $G \in R^{\mathrm{n}}$ is the Coriolis force and centrifugal force vector, $d \in R^{\mathrm{n}}$ is the joint friction vector, $G \in R^{\mathrm{n}}$ is the gravity vector, $\tau_{a} \in R^{\mathrm{n}}$ is the joint driving torque vector, and $\tau_{e x t} \in R^{\mathrm{n}}$ is the contact torque vector of the manipulator in the process of performing tasks. The simplified equation can be expressed as:

$$
\begin{aligned}
& \tau_{a}=\tau+\eta \\
& \eta \triangleq C(\mathrm{q}, \dot{q})+\mathrm{d}(\mathrm{q}, \dot{q})+\mathrm{G}(\mathrm{q})
\end{aligned}
$$

Equation (1) can be considered as the implicit feedforward and $\tau \in R^{\mathrm{n}}$ is used as the input:

$$
\mathrm{M}(\mathrm{q}) \ddot{q}=\tau+\tau_{\text {ext }}
$$

Generally, the movement of the manipulator is controlled by the end effector, and the specific work is carried out in the operation space. Compared with the three-dimensional space of the joint, the research on the controller will become more meaningful [14]. If the manipulator moves in the M- dimensional space, the Cartesian pose can describe the end effector as the M-dimensional vector about its coordinate axis/angle. Cartesian velocity $\dot{\boldsymbol{X}}$ can be described as torsion and Cartesian acceleration $\ddot{\boldsymbol{X}}$ as its derivative. Therefore, the relationship between the manipulator with $\mathrm{n}$ degrees of freedom and its M-dimensional space is as follows:

$$
\begin{aligned}
& \dot{x}=J(\mathrm{q}) \dot{q}, J(\mathrm{q})=\frac{\partial k(\mathrm{q})}{\partial q} \\
& \ddot{x}=J(\mathrm{q}) \ddot{q}+h(\mathrm{q}, \dot{q}) \\
& \qquad(\mathrm{q}, \dot{q}) \triangleq J(\mathrm{q}, \dot{q}) \dot{q}
\end{aligned}
$$

In the above equations, $k(\bullet): \mathrm{R}^{n} \rightarrow R^{m}$ is positive kinematics and $\boldsymbol{J} \in \boldsymbol{R}^{m^{* n}}$ is the Jacobian matrix, which maps the joint velocity to the Cartesian linear/angular velocity of the end of the manipulator. For redundant manipulator, when $\mathrm{n}>$ $\mathrm{m}$ and $\mathrm{r}=\mathrm{n}-\mathrm{m}$, the $\mathrm{r}$-dimensional vector is needed to describe the zero space of Jacobian matrix J [15]. The velocity vector $\dot{\boldsymbol{x}}_{N}=\boldsymbol{J}_{N}$ (q) $\dot{\boldsymbol{q}}$ in the r-dimensional zero space can rewrite equation (5) with the extended Jacobian matrix $J_{E}(\mathbf{q}) \in \mathbf{R}^{m^{* m}}$ included:

$$
\left(\begin{array}{c}
\dot{x} \\
\dot{x_{N}}
\end{array}\right)=J_{E}(\mathrm{q}) \dot{q}=\left(\begin{array}{l}
J(\mathrm{q}) \\
J_{N}(\mathrm{q})
\end{array}\right) \dot{q}
$$

$J_{N} \in \mathbf{R}^{m^{*} m}$ is the zero space Jacobian matrix.

$\ddot{X}$ is used to rewrite equation (6):

$$
\ddot{q}=J^{+}(\ddot{x}-h)+\mathrm{N}^{T} \lambda
$$

$J^{+} \in R^{m^{*} m}$ is the pseudo inverse matrix of J, and $N \in R^{\mathrm{n}^{*} m}$ is the zero-space basis matrix of J.

The equation is $J(\mathrm{q}) \mathrm{N}(\mathrm{q})^{T}=0, \lambda \in R^{m^{*} m}$, which is a vector whose value does not affect the Cartesian space motion, but can be used to achieve zero space motion as expected. It can be put into equation (4), and the expression is as follows:

$$
\tau=\operatorname{MJ}^{+}[\ddot{x}-\mathrm{h}]+\mathrm{MN}^{T} \lambda-\tau_{e x t}
$$

The force Jacobian relation $\tau_{e}=\boldsymbol{J}^{T} \boldsymbol{f}$ is used in the equation, and $\mathrm{f}$ is the contact force acting on the end effector: 


$$
\tau=\mathrm{MJ}^{+}[\ddot{\bar{x}}-\mathrm{h}]+\mathrm{MN}^{T} \lambda-J^{T} f
$$

The main purpose of introducing the Cartesian position inner loop is to improve the execution precision and anti-interference ability of the actuator as much as possible:

$$
\ddot{x}=\ddot{x}+k_{D} \tilde{x}+k_{p} \tilde{x}
$$

$\boldsymbol{x} \in \boldsymbol{R}^{m}$ is the difference between the desired position and the actual position in Cartesian space,

and $x \in R^{m}$ is the difference between the desired velocity and the actual velocity in Cartesian space, and $k_{p}$ and $k_{D}$ are the gain of the inner position loop. They are put into equation (12), and the expression is as follows:

$$
\tau=\operatorname{MJ}^{+}[\ddot{x}-\mathrm{h}]+\mathrm{MN}^{T} \lambda-J^{T} f
$$

For the 7-DOF redundant manipulator ( $\mathrm{n}=7$ and $M=6$, i.e., the redundant degree of freedom is 1 ), the control method uses the position inner loop to realize the Cartesian space motion control of the end effector. Compared with the general inverse dynamics control method, it has higher position tracking accuracy of the end effector [16]. The input $\lambda$ is used to ensure the normal self-motion of the manipulator without being affected by the end effector [17].

\subsection{Self-motion Obstacle Avoidance of 7-DOF Redundant Manipulator}

\section{A. Collision detection}

When the closed arm plane composed of the shoulder joint, elbow and wrist is tangent to the obstacle, there are only two cases between the robot arm and the obstacle, one is a collision, and the other is no collision. And then the artificial potential field method is introduced to further detect the robot arm, obstacles, and the virtual repulsion force acting on the vertical direction of the robot arm plane [18].

The normal number $d_{\text {safe }}$ represents the safe distance to be kept from the obstacle when the manipulator is moving, and $\mathrm{d}_{\lim }$ is the limit distance, which should meet the requirements of $d_{\text {safe }}>d_{\text {lim }}$. And the data in the space where the manipulator is located can be obtained by the camera. Subsequently, the surrounding shell can be obtained, which is composed of two equipotential surfaces of the potential field of obstacles [19]. D is the minimum safe distance between the plane of the manipulator and the surface of the obstacle. The distance between the manipulator and the obstacle is shown in Figure 3:

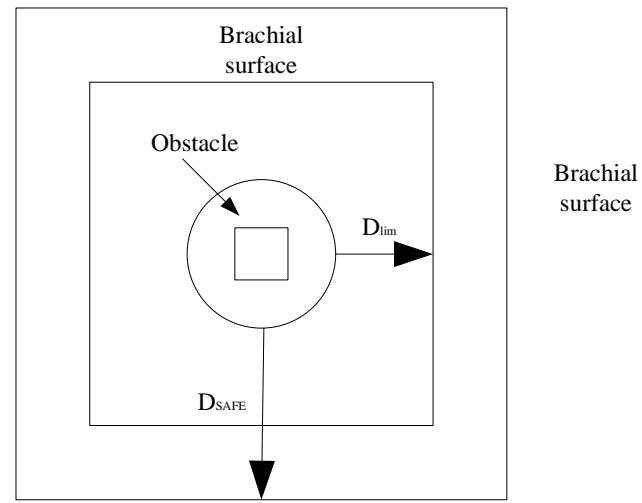

Figure 3 Distance between plane and obstacle

The virtual repulsive force is calculated based on $\mathrm{D}$, and $\mathrm{d}_{\text {safe }}$ and $\mathrm{d}_{\mathrm{lim}}$ are used to calculate virtual repulsion force:

$$
f_{v}= \begin{cases}0 & \mathrm{~d}>\mathrm{d}_{\text {safe }} \\ K\left(\frac{1}{d}-\frac{1}{d_{\text {safe }}}\right) & \mathrm{d}_{\text {lim }}<d<d_{\text {safe }} \\ f_{\max } & \mathrm{d}<\mathrm{d}_{\lim }\end{cases}
$$

In the above equation, $\mathrm{F}_{\max }$ is the maximum value of virtual repulsion force and $K$ is the proportional coefficient:

$$
K=f_{\max } \frac{d_{\text {safe }}-d_{\text {lim }}}{d_{\text {safe }} d_{\lim }}
$$

Equation (20) is used to protect the manipulator in two ways. First, when the manipulator is close to the obstacles during the movement and $\mathrm{D}$ is less than

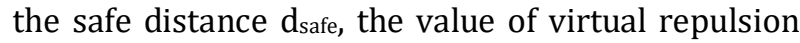
force is continuously changed between 0 and $F_{\text {max. }}$ Second, when the manipulator is further close to the obstacle and D is less than the limit distance $\mathrm{d}_{\text {lim, }}$ the virtual rejection force and $F_{V}$ value are constantly equal to $F_{\max }$. In this case, the system is considered in an emergency state, and the movement speed of the actuator of the manipulator needs to slow down and even stop until the manipulator is out of danger [20]. In view of more than one obstacle in the workspace, virtual repulsion is studied, and $\mathrm{v}$ is expressed as the sum of repulsive forces required by all obstacles, namely:

$$
f_{v}=\sum_{n} f_{v n}
$$

$\mathrm{n}$ is the total number of all obstacles in the operation of the manipulator and $f_{\mathrm{VN}}$ is the virtual repulsive force required by the $n$-th obstacle. 


\section{B. Self-motion obstacle avoidance}

To smoothly control the self-motion of the manipulator under the action of a virtual repulsive force, the following second-order linear differential equation is defined:

$$
M_{N} \tilde{x}_{N}+D_{N} x_{N} \tilde{=} f_{v}
$$

In the above equation, $M_{N}$ is the inertia coefficient, $D_{N}$ is the damping coefficient, and, they

are positive. The virtual repulsive force $x_{N}$ and its

derivative $x_{N}$ can be obtained by integrating the virtual repulsive force $f_{v}$. The equation similar to

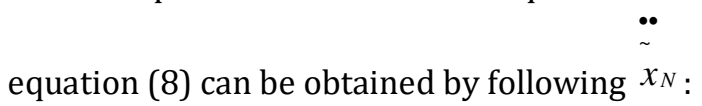

$$
\ddot{q_{N}}=N^{T}\left[\mathrm{M}_{N}^{-1}\left(\mathrm{f}_{v}-\mathrm{D}_{N} \tilde{x_{N}}\right)-h_{N}\right]
$$

The Equation below is obtained:

$$
\mathrm{h}_{N} \triangleq J_{N} \dot{q_{N}}
$$

Then, equation (19) is put into equation (9) to obtain the new expected angular acceleration:

$$
\ddot{q}=J^{+}(\ddot{x}-\mathrm{h})+\mathrm{N}^{T}\left[\mathrm{M}_{N}^{-1}\left(\mathrm{f}_{v}-\mathrm{D}_{N} \tilde{x_{N}}\right)-\mathrm{h}_{N}\right]
$$

Finally, the above equation is used to modify equation (13), the followings are obtained:

$$
\begin{aligned}
& \tau=M J^{+}\left[\ddot{x}^{*}-\mathrm{h}\right]-\mathrm{J}^{T} f+ \\
& M N^{T}\left[\mathrm{M}_{N}^{-1}\left(\mathrm{f}_{v}-\mathrm{D}_{N} \ddot{x}_{N}\right)-\mathrm{h}_{N}\right]
\end{aligned}
$$

It should be specially pointed out that in case of emergency, when $\mathrm{D}$ is less than the limit distance $\mathrm{d}_{\mathrm{lim}}, \mathrm{K}_{\mathrm{P}}$ and $\mathrm{K}_{\mathrm{D}}$ of the position inner loop can be set smaller or 0 to reduce the stiffness of the end effector and the moving speed of the manipulator.

\subsection{Design of Experiment Platform}

To test the method of avoiding obstacles for manipulators in self-motion, the self-maintenance platform on orbit is established to complete the

\begin{tabular}{|c|c|c|}
\hline $\begin{array}{c}\text { CR } \\
\text { number }\end{array}$ & $\mathrm{L} / \mathrm{m}$ & $\mathrm{M} / \mathrm{kg} \& \mathrm{~J} /\left(\mathrm{kg} \cdot \mathrm{m}^{2}\right)$ \\
\hline A & 0.09 & $3.44(0.011,0.010,0.110)$ \\
\hline B & 0.18 & $2.26(0.018,0.023,0.025)$ \\
\hline $\mathrm{C}$ & 0.18 & $2.28(0.002,0.006,0.008)$ \\
\hline $\mathrm{D}$ & 0.15 & $1.82(0.002,0.046,0.046)$ \\
\hline $\mathrm{E}$ & 0.15 & $1.75(0.004,0.003,0.004)$ \\
\hline $\mathrm{F}$ & 0.15 & $1.91(0.003,0.006,0.008)$ \\
\hline
\end{tabular}
experimental research. The research object is fiveaxis redundant industrial manipulator. The data of the specific joint parts are shown in Table 1:
Table 1 Arm parameters of 7-DOF redundant manipulator

The other important control parameters of the manipulator are $\mathrm{k}=6.00 \mathrm{~N} / \mathrm{m}, \mathrm{d}_{\text {sale }}=0.05 \mathrm{~m}$ and $\mathrm{d}_{\text {lim }}=$ $0.01 \mathrm{M}$.

The mechanical and electrical integration method is adopted for the design of the mechanical arm joint. The brushless motor drives the mechanical arm, and transmits the information of the accurate position and rotation angle of each joint continuously. A PCI104 computer communicates with the FPGA controller integrated into the joint through the PPSeCo bus, and the communication cycle is $1 \mathrm{~ms}$. The computer with the dual-core processor is used to complete the closed-loop control of the manipulator and a series of calculations, and the data transportation is realized through PCI bus [21, 22]. The most advanced NIOS processor is used for the joint controller. The computer sends instructions to control the motor, thereby realizing system control. Meantime, the data received from the sensor are returned to the computer again, as shown in Figure 4:

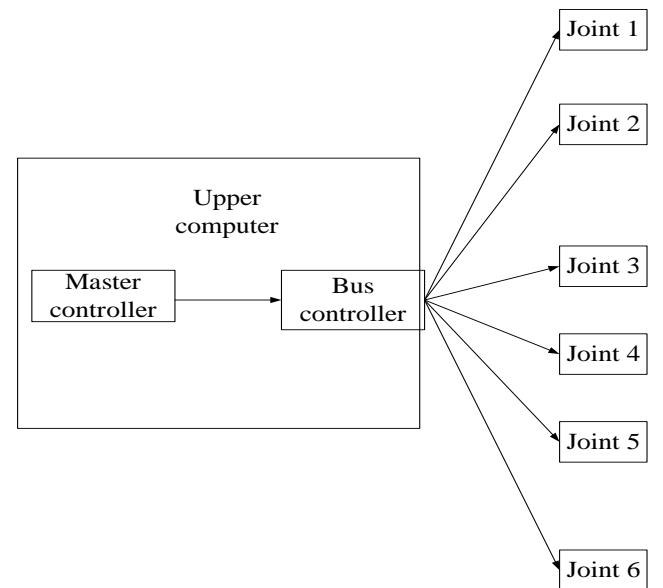

Figure 4 Construction of experimental platform

\section{Experimental Analysis and Simulation Research \\ 3.1 Motion Simulation of Manipulator}

The above methods are adopted to obtain the motion data of the manipulator, which are calculated, analysed and transmitted to the control processor, helping control the servo mechanism for accurate motion. 
The trajectory planning of the manipulator's joint in three-dimensional space mainly includes cubic and quintic polynomial interpolation planning and spline function planning. The polynomial interpolation method is used to confirm the trajectory between the manipulator and the destination, from the starting point to the end point.
The quintic polynomial interpolation function step5 built in ADAMS software is used for trajectory planning.

The data are imported into ADAMS software to simulate the trajectory of the manipulator. The first simulation steps are 90 . The simulation results are shown in Figure 5-7:

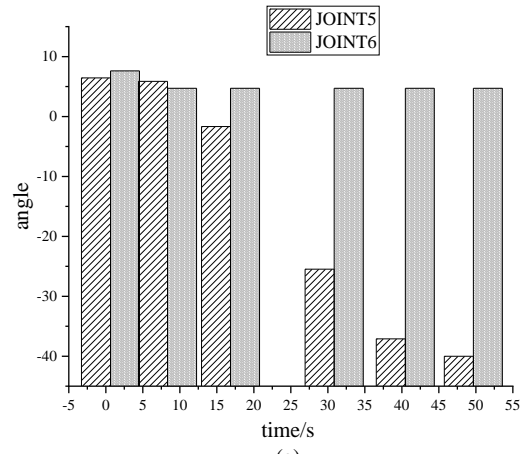

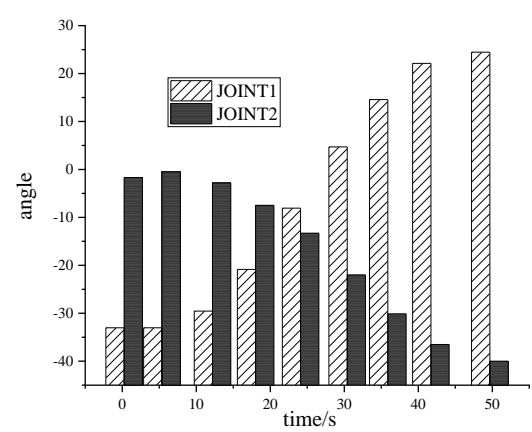

(b)

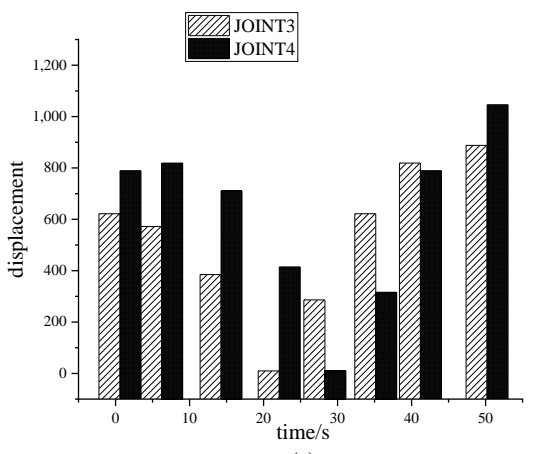

(c)

Figure 5: Joint displacement curve (a-c is the displacement and angle change curve of joints 1-6)
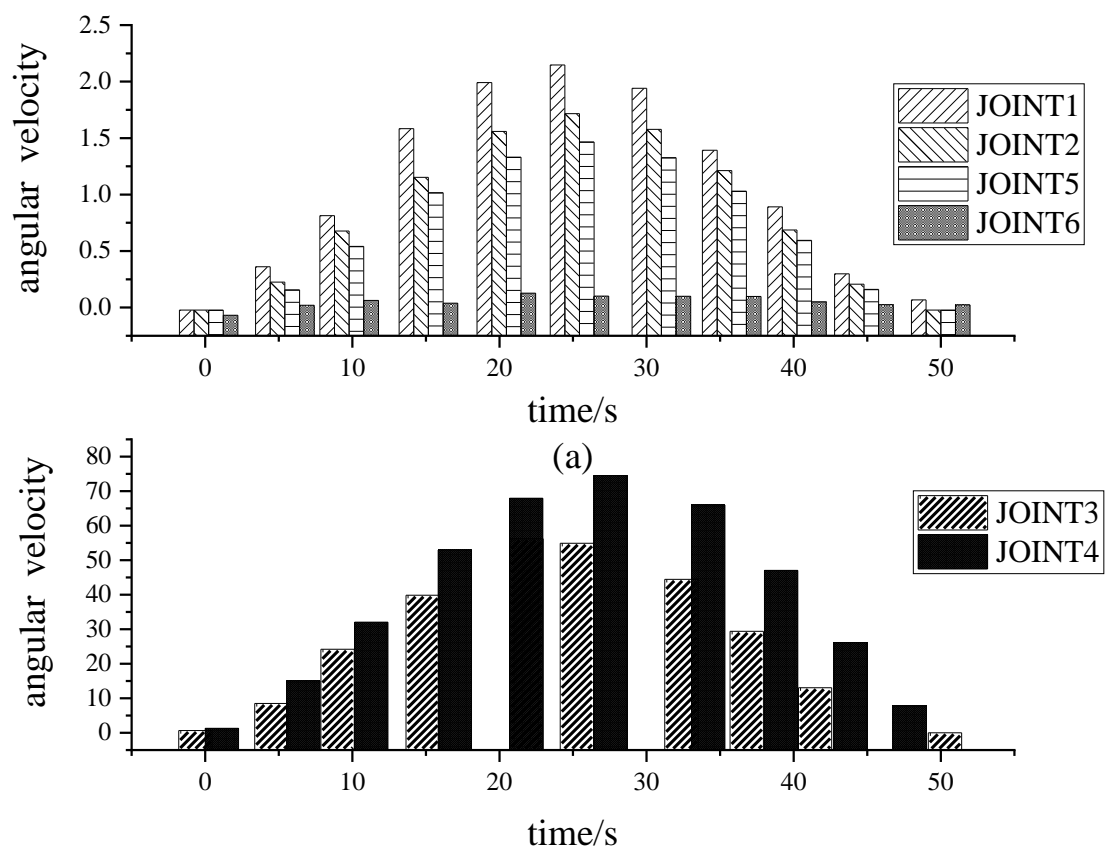

(b)

Figure 6: Joint speed curve (Figure (a) and (b) show angular velocity and angle change curve of joints 1-6 respectively) 

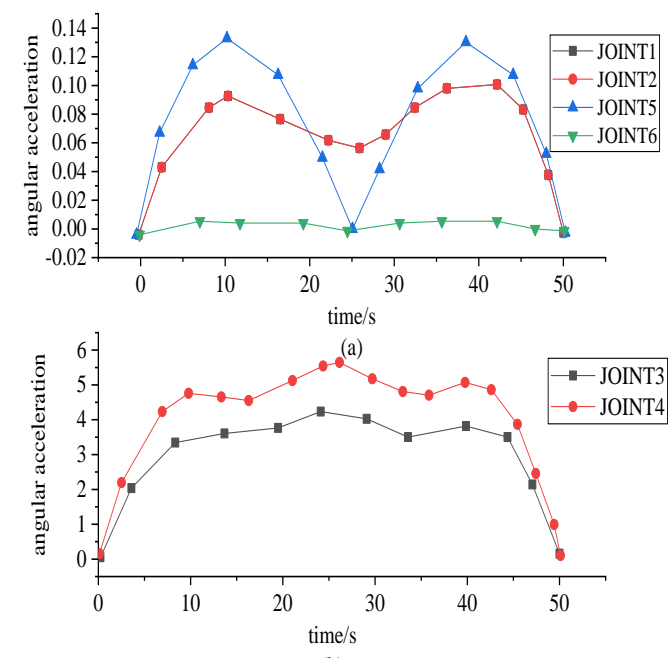

(b)

Figure 7: Joint acceleration curve ((a) and (b) are the time angular velocity curve of 1-6 joints)

Figure 5 is the displacement curve of each joint of the manipulator, from which the change of each joint can be observed. 1, 2, 5 and 6 belong to rotational joints, which rotate around the Z-axis in threedimensional space, and 3 and 4 are horizontal moving joints, which mainly show plane movement.
Figure 5 shows the angular velocity curves of rotating joints $1,2,5$ and 6 , and the velocity curves of moving joints 3 and 4 . Figure 6 indicates the angular acceleration curves of the rotating joints 1,2 , 5 and 6 , and the acceleration curves of moving joints 3 and 4 . There is no obvious mutation point in each joint.

Figure. 5, Figure 6 and Figure 7 are compared, and it can be found that there is little difference between the changes of joints 5 and 6 . This is because $\theta_{6}$ is determined by ${ }^{\theta_{2}}$ and $\theta_{5}$ together in the calculation, while in the process of moving, the change of $\theta_{5}$ is much greater than that of $\theta_{2}$, and the effect on $\theta_{2}$ is much less than that of $\theta_{5}$. As a result, the change curves of joints 5 and 6 are the same. Moving joints 3 and 4 change with their maximum displacement. Therefore, the change of joints 3 and 4 is little, and the total change of the smallest joint in all joints is reached.

\subsection{Simulation Analysis of Obstacle Avoidance of Manipulator}

The change curve of the mass-damping experiment (M: mass; D: damping) is shown in Figure 8.
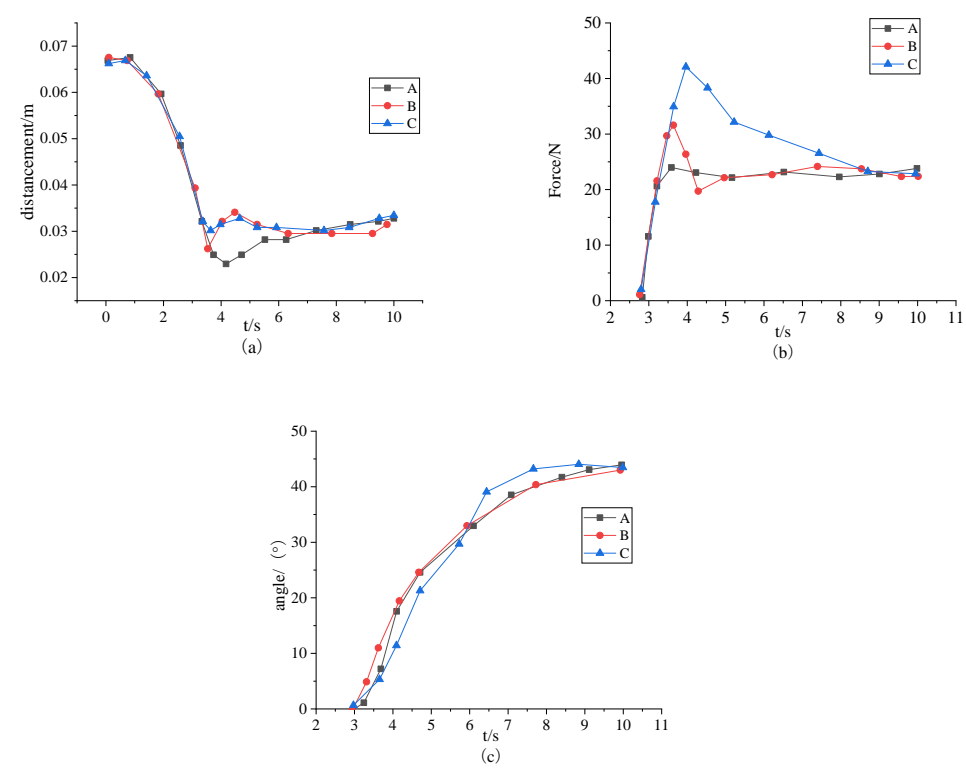

Figure 8: Change curve of mass-damping experiment (a: distance change between manipulator and obstacles; b: virtual repulsive force; : arm angle; $A: M=0.2, D=1 ; B: M=0.2, D=2 ; C: M=0, D=1$ )

The experimental results show that the safest distance between the manipulator and the obstacle is $71 \mathrm{~mm}$. When the end actuator is started, the manipulator will move to the obstacle. When the manipulator is moving for four seconds, the minimum safety distance between the manipulator and the obstacle reaches $50 \mathrm{~mm}$. The manipulator is still moving and near the obstacles because of the inertia of the mass-damping system of the manipulator. And then the motion speed of the manipulator is gradually driven by the virtual rejection force, and slows down and even stops before the obstacle with a safety distance of $39 \mathrm{~mm}$ between the last arm and the obstacle (Figure 8 (a)). When the whole system is within a safe distance, the manipulator will generate a repulsive force on the equipotential plane of the artificial potential field, thus preventing it from approaching the obstacles. 
When the distance between the manipulator and the obstacle is the closest, the relation between the manipulator and the obstacle will be linear and finally stabilized at 24N (Figure 8 (b)). With the help of the repulsive force, the plane of the arm is twisted towards the obstacle avoidance surface, producing the self-motion of the manipulator. To avoid obstacles, the plane of the arm is turned $39^{\circ}$ during the movement of the manipulator (Figure 8 (c)).

The dynamic difference of the end-effector position is less than $9 \mathrm{~mm}$, and the static difference is less than $3 \mathrm{~mm}$. The differences can be mainly attributed to the following: the slight difference in the setting of the parameters of the manipulator's arm, and the flexibility of each joint itself.

\section{Discussion}

The manipulator has seven degrees of freedom, and the kinematics model is established with reference to the manipulator motion principle [23]. Based on the established kinematics model, the forward and inverse kinematics equations of the manipulator are obtained, and the moving process of the manipulator is simulated in Matlab software. Then, the rationality of the equations is verified, and the optimal trajectory of the manipulator is set. The virtual model of the manipulator is established in Simulink, and the simulation experiment and analysis of the model are carried out. From the change curve obtained from the simulation test, the motion path of the manipulator tends to be smooth, and can avoid obstacles completely [24].

During the working process, the manipulator needs to avoid obstacles and large contact as far as possible to complete the tasks. This experimental study shows that the manipulator can fully realize the expected goal [25]. The manipulator can perform smooth self-motion and keep a certain safe distance between the virtual repulsive force and the obstacle. The change curve proves that the relation between the virtual repulsive force and the motion displacement is linear. Therefore, the conclusion can be drawn: the manipulator always keeps a safe distance from the obstacle and performs the tasks successfully. In the completion of the task, the end effector is not subjected to the influence of the movement of the manipulator [26].

\section{Conclusion}

The study is carried out by applying to the designed manipulator, which is composed of seven degrees of freedom. Based on the characteristics of the manipulator and the workspace, the motion model is established to simplify the inverse motion solution process, and meet the requirements of the motion trajectory of the manipulator. The orbit simulation of joints in three-dimensional space is conducted by using the method of the quintic polynomial. Moreover, the curves of displacement, acceleration and angular velocity of these joints are analyzed. The changes of the curve show that the joints are moving smoothly and there is no serious track offset. Therefore, the control system used to operate the joint based on the motion principle of the composite manipulator is feasible. However, the study also has some shortcomings: (1) in the simulation collision process of the manipulator, the measured object is regarded as a whole, and the details are neglected, leading to inaccurate measurement; (2) the motion curve shows that the area of the motion path is smooth and there is no big fluctuation. However, the arm angles of each joint of the manipulator are easy to change with the surroundings; (3) the real-time online path planning test of the manipulator is not completed. The simulation path tests for the manipulator in the process of obstacle avoidance and trajectory planning need to be studied further.

\section{Acknowledgment}

This work was supported by Supported by Teacher Key University for Foundation of Province Henan Research (Project Number:2019GGJS295), Key Specialized Research and Development Breakthrough in Province (Project Number:182102210084) and Science and Technology Innovation Project of Xinlian College of Henan Normal University (Project Number: 2018XLXYCXTD-001).

\section{References}

[1] Zhao W. F., Li X. X., Chen X, et al. (2020) Bicriteria Acceleration Level Obstacle Avoidance of Redundant Manipulator. Frontiers in neurorobotics, 14, 54-54.

[2] Benzaoui M., Chekireb H., Tadjine M, et al. (2016) Trajectory tracking with obstacle avoidance of redundant manipulator based on fuzzy inference systems. Neurocomputing, 196, 23-30.

[3] Li C., Ma Y., Zhang Y, et al. (2020) Obstacle Avoidance and Multitarget Tracking of a Super Redundant Modular Manipulator Based on Bezier Curve and Particle Swarm Optimization.Chinese Journal of Mechanical Engineering, 33(05), 111129.

[4] David C. O., Isaac C., Alexander P. (2020) Robust control for master-slave manipulator system avoiding obstacle collision under restricted working space. IET Control Theory \& Applications, 14(10), 1375-1386.

[5] Wei D. W., Gao T., Mo X. J, et al. (2020) Flexible Bio-tensegrity Manipulator with Multi-degree of Freedom and Variable Structure. Chinese Journal of Mechanical Engineering, 33(1), 3.

[6] Nithya P. K., Priya P. S., Gifty E. B, et al. (2020) Optimal Path Planning and Static Obstacle 
Avoidance for a Dual Arm Manipulator Used in On-Orbit Satellite Servicing. IFAC Papers OnLine, 53(1), 189-194.

[7] Meiringer M., Kugi A., Kemmetmüller W. (2019) Time-optimal fold out of large-scale manipulators with obstacle avoidance. IFAC PapersOnLine, 52(16), 114-119.

[8] Xu Z. H., Zhou X. F., Li S. (2019) Deep Recurrent Neural Networks Based Obstacle Avoidance Control for Redundant Manipulators. Frontiers in neurorobotics, 13, 47.

[9] Sha H. B., Ghariblu B. (2019) Obstacle avoidance of redundant robotic manipulators using safety ring concept. International Journal of Computer Integrated Manufacturing, 32(7), 695-704.

[10] Zhou X. F., Xu Z. H., Li S. Collision-Free Compliance Control for Redundant Manipulators: An Optimization Case. Frontiers in Neurorobotics, 13,50

[11] Kang G., Kim B. Y., Lee Y. H, et al. (2019) Sampling-based motion planning of manipulator with goal-oriented sampling. Intelligent Service Robotics, 12(3), 265-273.

[12] Liao J. F., Huang F. H., Chen Z, et al. (2019) Optimization-based motion planning of mobile manipulator with high degree of kinematic redundancy. International Journal of Intelligent Robotics and Applications, 3(2), 115-130.

[13] Xu J. J., Liu Z. F., Zhao Y. S, et al. (2019) A Path Optimization Technique with Obstacle Avoidance for an 8-DOF Robot in Bolt Looseness Detection Task. International Journal of Precision Engineering and Manufacturing, 20(5), 717-735.

[14] Jiang A., Yao X., Zhou J. (2018) Research on path planning of real-time obstacle avoidance of mechanical arm based on genetic algorithm. The Journal of Engineering, 16, 1579-1586.

[15] Dina Y. K., Pere R. (2018) Motion planning survey for autonomous mobile manipulators underwater manipulator case study. Robotics and Autonomous Systems, 107, 20-44.

[16] Satish C., Hari K. V. (2018) Trajectory Planning of Redundant Manipulators Moving Along Constrained Path and Avoiding Obstacles. Procedia Computer Science, 133, 627-634.
[17] Ram R. V., Pathak P. M., Junco S. J. (2018) Inverse kinematics of mobile manipulator using bidirectional particle swarm optimization by manipulator decoupling. Mechanism and Machine Theory, 131, 385-405.

[18] Guo D. S., Xu F., Yan L. C, et al. (2018) A New Noise-Tolerant Obstacle Avoidance Scheme for Motion Planning of Redundant Robot Manipulators. Frontiers in neurorobotics, 12, 51.

[19] Zong L. J., Luo J. J., Wang M. M, et al. (2018) Obstacle avoidance handling and mixed integer predictive control for space robots. Advances in Space Research, 61(8), 1997-2009.

[20] Arif A., Qassar A. L., Ali N. A. (2018) Optimal Path Planning Obstacle Avoidance of Robot Manipulator System using Bézier Curve. American Scientific Research Journal for Engineering, Technology, and Sciences (ASRJETS), 40(1), 6-17.

[21] Han D., Nie H., Chen J. B, et al. (2018) Dynamic obstacle avoidance for manipulators using distance calculation and discrete detection. Robotics and Computer Integrated Manufacturing, 49, 98-104.

[22] Yang H. J., Li L. J., Gao Z. J. (2017) Obstacle avoidance path planning of hybrid harvesting manipulator based on joint configuration space. Editorial Office of Transactions of the Chinese Society of Agricultural Engineering, 33(4), 55-62.

[23] Korayem M. H., Nekoo S. R. (2016) The SDRE control of mobile base cooperative manipulators: Collision free path planning and moving obstacle avoidance. Robotics and Autonomous Systems, 86. 86-105.

[24] Zhou D. S., Wang L., Zhang Q. (2016) Obstacle avoidance planning of space manipulator endeffector based on improved ant colony algorithm. Springer Plus, 5(1). 1-13.

[25] Sreekumar M. (2016) A Robot Manipulator with Adaptive Fuzzy Controller in Obstacle Avoidance. Journal of The Institution of Engineers (India): Series C, 97(3), 469-478.

[26] Benzaoui M., Chekireb H., Tadjine M, et al. (2016) Trajectory tracking with obstacle avoidance of redundant manipulator based on fuzzy inference systems. Neurocomputing, 196, 23-30. 